STRUCTURAL SCIENCE CRYSTAL ENGINEERING MATERIALS

ISSN 2052-5206

Keywords: book review; mineralogy.

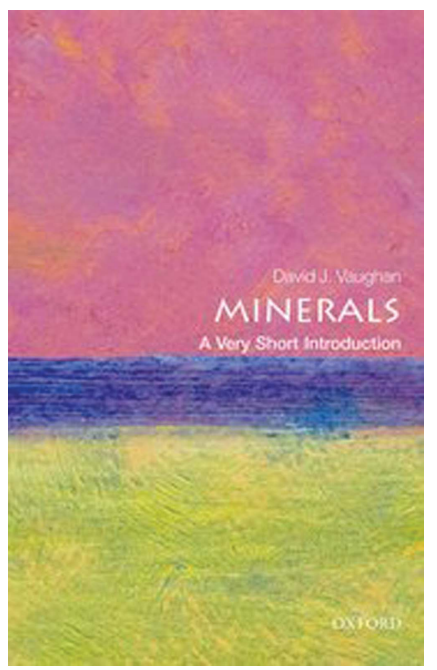

(C) 2016 International Union of Crystallography

\section{Minerals: A Very Short Introduction. By David J. Vaughan. Oxford University Press, 2014. Paperback, Pp. 160. Price GBP 7.99. ISBN 9780199682843.}

\author{
Cristiano Ferraris* \\ Laboratoire de Minéralogie - USM0201, Muséum National d'Histoire Naturelle, 61 rue Buffon, Paris 7500, France. \\ *Correspondence e-mail: ferraris@mnhn.fr
}

Nowadays, interest in minerals is widespread and the results of mineral sciences are a necessary background not only in classical Earth science but also in materials, biological, forensic, geophysical and other branches of sciences. The author is well known for his 'molecular scale studies of mineral surfaces including interactions with microbial species, and applications of such studies to problems of Earth resources (including mineral extraction technologies) and the environment', as one can read on the web site of the School of Earth, Atmospheric and Environmental Sciences at the University of Manchester (http://www.seaes.manchester.ac.uk/people/staff/profile/?ea=david. vaughan).

In this pocket book David J. Vaughan has done an outstanding job presenting complex notions in simple terms and providing examples to which the reader can relate. Following his research interests, he combines classic mineralogy together with aspects such as the role of minerals in the living world and environment, going through modern aspects of analytical methods. The output is an unconventional but very appealing presentation of mineralogy aimed at the amateurs and anyone interested in minerals, even if some basic knowledge of chemistry and physics is required. This introduction to mineralogy could also be a quick and easy lecture for those students looking for inspiration to focus their attention on different aspects of modern mineral sciences.

The book, well illustrated with greyscale drawings and images, is divided into seven chapters, and throughout terms are defined in an exhaustive way. Unfortunately, any colour image is used to better illustrate thin section photos, crystal structures and geological sketches; the quality of the book would have been improved using a few colour images.

The first chapter presents the basics of mineralogy and crystallography. It serves as a quick refresher if the reader's notions of mineralogy are rusty.

Chapter 2 reviews analytical methods currently used to characterize mineral species, going from the principle of classic polarizing microscopy to more recent and powerful techniques, such as electron microscopy; some principles of X-ray diffraction and spectroscopy are also refreshed.

Chapter 3 is a very short course on geodynamics, relating minerals to their genesis and their impact on different tectonic environments; a short part is dedicated to high-pressure experiments that allow scientists to understand the behaviour of minerals under conditions not directly accessible, such as those occurring in the Earth's interior.

Chapter 4 introduces the cycling of minerals at, or very near, the surface of the Earth, particularly under weathering conditions. This is used as a starting point to present the latest frontiers of mineral sciences touching biological and environmental aspects, such as the role of nano-particles, nano-minerals and aerosols, including their peculiar physical and chemical properties in terms of surface and interface interactions.

Chapter 5 approaches other very relevant and fashionable arguments, such as biominerals and bio-mineralogy. The author's approach is extremely clear and the descriptions of how the biological world (organisms) can produce mineral phases not belonging to the classic geological processes are clearly defined.

Chapter 6 discusses minerals as resources, taking into consideration all the major aspects of different mineral sources and properties, how mineral deposits form and how and why these resources are exploited. 


\section{book reviews}

Chapter 7 resumes a frontier topic of mineralogy: the mineral evolution starting from the origin of the solar system until anthropic causes going through different stages related to our planet's evolution such as the appearance of water and oxygen up to the emergence of life. The chapter ends with a digression on the mineral resources for the present and, as far as it is possible, the future.
Last but not least the author presents a very useful list of further reading for those readers that would like to go one step further to a better understanding of more complex mineralogical subjects.

Overall I highly recommend this book, even if one must be clear that it is no more than a short, even if quite stimulating, introduction to the mineral world. 